P136 (continued)

Rationale: Physical activity and nutrition are influential factors for childhood obesity and Type 2 Diabetes. Maryland adolescents typically get fewer than the recommended 60 minutes of physical activity daily and do not eat the recommended amounts of fruits and vegetables. Improving access to healthy foods in schools and increasing availability of active time can potentially influence the development of these chronic conditions.

Description: The Healthy Eating Active Lifestyle (HEAL) coalition created a Healthy Schools Challenge to promote healthy eating, physical activity and overall wellness with students. It is open to all public schools in Washington County. The challenge includes three categories: Fuel Your Body (nutrition), Get Moving (physical activity) and Feel Your Best (mental health). Criteria is based on best practices from the Alliance for a Healthier Generation. Schools select options in each category to earn points. Schools can earn a "healthy school status" as well as prize money based on their points accumulation. At the end of the school year, schools that go above and beyond in creating a a healthier school environment are recognized.

Evaluation: Both teacher and student evaluations are completed to assess changes in school environments and behavior changes.

Conclusions and Implications: Competition promotes healthy schools as our second year of the Healthy Schools Challenge created more activity and innovation. This challenge can be easily adapted in other communities.

Funding: HEAL of Washington County

\section{P137 Knowledge, Attitudes, and Self-Efficacy of Culinary Skills as they Relate to Chronic Disease Prevention in Future Health Practitioners: iCHOP Pilot Study}

Rebecca Hagedorn, BS, West Virginia University; Allison Morris, BS; Rashel Clark, BS, RDN; Britteny Harris, BS; Ashley George, BS; Joseph Enfonde, BS; Donald Brubaker, BS; Melissa Olfert, DPH, DrPH, RDN, melissa.olfert@mail.wvu. edu, West Virginia University, 333 AG SC Building G28, Morgantown, WV 26506

Objective: Nutrition and culinary preparation of food, is a key component of disease prevention and treatment. However, nutrition education for health professional students is often minimal or absent. iCHOP Pilot Study investigated knowledge, attitudes and self-efficacy of nutrition and culinary skills in specific disease states of medical students at West Virginia University.

Study Design: After IRB approval, medical students, $\sim 400$, were emailed a 45 items survey $(\sim 15$ $\mathrm{min}$ ) focused on diabetes, cardiovascular disease, and pregnancy investigating their nutrition and culinary knowl- edge (multiple choice), attitudes (Likert 5 pt), and selfefficacy across disease states.

Results: Participants $(n=56)$ attitudes of importance of nutrition in medicine addressing the role of diet with a patient strongly agree (87.5\%), physicians should counsel high-risk patients about nutrition $(89.6 \%$ agreed or strongly agreed). Nutrition knowledge was overall low (62.5\% of correct responses) of disease topics resulted with pregnancy scoring lowest $(56.4 \%)$, diabetes (58.1\%) and cardiovascular disease (73.1\%). Similarly, self-efficacy was low with pregnancy self-efficacy to advise a patient on breastmilk vs other options $(67.2 \%$ of students stated not feeling proficient) $46.6 \%$ stated not proficient in nutrition education for cardiovascular disease and $48.3 \%$ stated somewhat proficient in weight loss aspects in patients with diabetes. Participants reported the highest self-efficacy (62.1\% totally proficient) on benefits of exercise on health.

Conclusion: Nutrition, food preparation, including culinary skills, should be incorporated in training and continuing education curriculum components of health professions including those in medicine.

Funding: $\mathrm{NIH}$

\section{P138 Nutrition Education for Cognitive Impaired Audiences}

Sheilah Hebert, MS, RD, IBCLC, hebertsh@anr.msu.edu, Michigan State University, 446 West Circle Drive, Room 250, East Lansing, MI 48824; Diane Rellinger, MS, Michigan State University

Objective: Individuals with developmental disabilities and cognitive impairments have poor eating habits and are at increased risk for cardiovascular disease, obesity, and osteoporosis. We have found that there is a great need for a curriculum to serve the special needs of this population in Michigan.

Description: This curriculum was piloted with 120 people. The pilot sites include urban school settings, urban community settings as well as rural community sites. Eight lessons were delivered which included both food tasting and cooking. The target audience was adults with development disabilities and cognitive impairment.

Theory, Prior Research, Rationale: A work group was convened to identify current needs for curriculum to provide nutrition and physical activity education to cognitively impaired audiences. This group developed a survey to be completed by agencies that service participants that are cognitively impaired. This survey identified the optimal topics to be covered along with the preferred format for the delivery of nutrition and physical activity content. A literature review was also completed to assess current practices used in providing services to this population. Based on this information Healthy Eating Adds Up was identified for piloting. Protocols as well as evaluation tools were developed to pilot this curriculum. Strategies to increase our outreach and 